DOI: $10.12957 /$ demetra.2016.21458

\title{
Indicadores antropométricos e fatores associados: um estudo em policiais civis de Vitória-ES, Brasil
}

\section{The anthropometric indicators and associated factors: a study in civilian police officers from Vitória-ES, Brazil}

\author{
Luciane Bresciani Salaroli \\ Renata Manoel2 \\ Miriani Delfino Botacim² \\ Glenda Blaser Petarli $i^{3}$ \\ Monica Cattafesta ${ }^{4}$ \\ Eliana Zandonade 5
1 Universidade Federal do Espírito Santo, Curso de Nutrição, Departamento de Educaçã̃o Integrada em Saúde, Programa de Pós- graduação em Nutrição e Saúde, Programa de Pós-graduação em Saúde Coletiva. Vitória-ES, Brasil.
${ }^{2}$ Universidade Federal do Espírito Santo, Curso de Nutrição, Departamento de Educaçãão Integrada em Saúde. Vitória-ES, Brasil.
${ }^{3}$ Universidade Federal do Espírito Santo, Hospital Universitário Cassiano Antônio de Moraes. Vitória-ES, Brasil.
${ }^{4}$ Universidade Federal do Espírito Santo, Curso de Nutrição, Departamento de Educação Integrada em Saúde, Programa de Pós-graduação em Nutrição e Saúde. Vitória-ES, Brasil.
${ }^{5}$ Universidade Federal do Espírito Santo Departamento de Estatística, Programa de Pós- graduação em Saúde Coletiva. Vitória-ES, Brasil.
Correspondência / Correspondence Luciane Bresciani Salaroli
E-mail: lucianebresciani@gmail.com

\section{Resumo}

A sociedade moderna tem passado por alterações nos padrões alimentares e comportamentais, influenciando negativamente a saúde do trabalhador, como no caso de policiais civis, contribuindo com o adoecimento e o comprometimento do estado nutricional neste grupo. O estudo teve como objetivo investigar indicadores antropométricos (Índice de Massa Corporal - IMC e Circunferência de Cintura - CC) e sua relação com fatores sociodemográficos, laborais e de hábitos alimentares em policiais civis de Vitória/ES. Utilizou-se metodologia quantitativa e transversal, com 133 policiais, com idade entre 25 e 65 anos, de ambos os sexos, que trabalhassem na região da Grande Vitória/ ES, selecionados de forma aleatória. Foram realizados testes qui-quadrado e regressão logística multivariada para as variáveis que apresentaram significância estatística de até 20\%. Constatouse alta prevalência de excesso de peso $(64,3 \%$; $\mathrm{n}=83)$, o IMC se relacionou com classe econômica elevada, sexo masculino, considerar o trabalho estressante e hábito de realizar café da manhã fora do lar, e a CC se associou à idade superior a 40 anos. Ressalta-se a importância da avaliação antropométrica em policiais civis, a fim de identificar fatores associados à inadequação do estado nutricional.

Palavras-chave: Polícia. Estado nutricional. Hábitos alimentares. Saúde do trabalhador. Condições de trabalho. 


\section{Abstract}

Modern society has undergone changes in dietary and behavioral patterns, negatively influencing the workers' health, as in the case of police officers, contributing to illness and endangering the nutritional status in this group. The study aimed to investigate the anthropometric indicators (body mass index - BMI and waist circumference - CC) and its relationship to sociodemographic factors, labor and eating habits in civilian police of Vitoria/ES. This is a cross-sectional study, with 133 policemen, aged between 25 and 65 years, of both sexes, who worked in the region of Grande Vitória/ES, randomly selected. A chi-square test and a multivariate logistic regression for variables which were statistically significant up to $20 \%$ were performed. A high prevalence of overweight was found $(64.3 \% ; \mathrm{n}=83)$, BMI was associated with high economic class, male, considering the stressful work and the habit of having breakfast outside the home and CC associated to the age of 40 years. The importance of anthropometric evaluation in civilian police is highlighted, in order to identify factors associated with inadequate nutritional status.

Key words: Police. Nutritional status. Food habits. Occupational health. Working conditions.

\section{Introdução}

No Brasil, as Doenças Crônicas não Transmissíveis (DCNT) foram responsáveis por 72,7\% dos óbitos no ano de 2011, com destaque para doenças cardiovasculares $(30,4 \%){ }^{1}$ Dentre os principais fatores de risco para as DCNT, ressaltam-se o sobrepeso e a obesidade, ${ }^{2}$ e neste cenário, segundo dados da Vigilância de Fatores de Risco e Proteção para Doenças Crônicas por Inquérito Telefônico (VIGITEL), 50,8\% da população brasileira apresentaram excesso de peso no ano de 2013, sendo 17,5\% considerados obesos. ${ }^{3}$ A mesma pesquisa revelou ainda que $49 \%$ da população adulta da cidade de Vitória/ES estavam acima do peso, e entre estes, 16,1\% encontravam-se obesos.

Em relação ao diagnóstico do estado nutricional, a utilização de indicadores antropométricos, como o Índice de Massa Corporal (IMC) e a Circunferência da Cintura (CC), é muito comum, sendo recomendada em estudos populacionais visto a simplicidade e o baixo custo dos métodos. ${ }^{4}$ Enquanto o IMC é amplamente usado na análise da presença de sobrepeso e obesidade, a CC, como medida isolada, é um importante preditor do tecido adiposo visceral, onde já é bem elucidada sua relação direta com doenças do aparelho cardiovascular. ${ }^{5}$ 
A relação entre a dieta desequilibrada, hábitos de vida poucos saudáveis e estado nutricional inadequado é bem estabelecida. ${ }^{6}$ Considerando que as pessoas passam a maior parte do tempo no local de trabalho e que as escolhas alimentares são definidas de acordo com o tempo, praticidade e disponibilidade alimentar, as características do trabalho podem influenciar na adoção de hábitos alimentares incorretos. ${ }^{7}$ Assim, muitas vezes os trabalhadores se sujeitam a uma alimentação prática e densamente calórica, contribuindo para prejuízos no estado nutricional e instalação de DCNT, além de outras comorbidades. ${ }^{8}$

Nesse contexto, tem-se buscado compreender as relações existentes entre o trabalho e a saúde do trabalhador, uma vez que as pressões e exigências impostas sobre os funcionários, aliadas às características psicológicas e estilo de vida pouco saudável, podem refletir em prejuízos na sua saúde física e mental. ${ }^{9}$ A exemplo disso, está a categoria de policial, considerada uma profissão que se destaca pelo elevado desgaste físico e mental decorrente da intensa carga de trabalho e sofrimento. ${ }^{10}$

São escassos na literatura estudos que investigam o estado nutricional e seus fatores associados em policiais. Os que se encontram disponíveis, em sua maioria, referem-se à classe dos policiais militares, ${ }^{11,12}$ que, por sua vez, apresentam particularidades de trabalho diferentes das do policial civil e não investigam a influência das variáveis relativas aos hábitos alimentares no perfil antropométrico dessa população. ${ }^{18}$

Dessa forma, buscando contribuir para o preenchimento dessa lacuna do conhecimento e tendo em vista a dinâmica do serviço policial, a relevância do condicionamento físico para o desenvolvimento de suas atividades e a expressiva importância para a sociedade desta categoria profissional, o objetivo deste estudo foi avaliar indicadores antropométricos e seus fatores associados em policiais civis de Vitória-ES.

\section{Metodologia}

Trata-se de um estudo quantitativo, transversal, desenvolvido a partir dos dados sociodemográficos, antropométricos, laborais e de hábitos alimentares de uma pesquisa mais ampla, realizada de janeiro a abril de 2014, intitulada "Estado nutricional, hábitos alimentares, risco cardiovascular e fatores associados: um estudo em policiais civis de Vitória/ES”. O trabalho foi submetido e aprovado pelo comitê de ética da UFES sob o número 20408013.8.0000.5060.

A população estudada foi constituída por policiais civis que trabalhassem na região da Grande Vitória com faixa etária de 25 a 65 anos de ambos os sexos. O tamanho amostral foi calculado considerando um número total de 1.225 policias, intervalo de confiança de $95 \%$, erro de $8 \%$, prevalência de excesso de peso de $50 \%$, totalizando uma amostra de 134 policiais. A participação no estudo se deu mediante assinatura do Termo de Consentimento Livre e Esclarecido (TCLE). A coleta de dados foi realizada no local de trabalho, sendo o funcionário liberado de suas funções 
no horário das entrevistas para responder a um questionário estruturado contendo questões sociodemográficas, laborais e sobre hábitos alimentares e para ser submetido à coleta de dados antropométricos por pesquisadores treinados. O critério de inclusão na pesquisa foi estar em plena atividade laboral, sendo excluídos gestantes, servidores em período de férias ou aposentados.

Em relação aos dados sociodemográficos, a classe socioeconômica foi determinada pelo Critério de Classificação Econômica do Brasil, ${ }^{13}$ os indivíduos foram categorizados em duas classes, A e B ou C, D e E, a fim de tornar os grupos representativos. A variável escolaridade foi categorizada em três grupos: ensino fundamental e médio, ensino superior e pós-graduação. O estado civil foi categorizado em solteiro, casado/vive com companheiro e separado/divorciado/viúvo.

Para a avaliação antropométrica, foram incluídas as variáveis: peso corporal, altura e circunferência da cintura (CC). O peso corporal foi aferido com auxílio da balança portátil Tanita ${ }^{\circledR}$ modelo digital BC533 InnerScan com precisão de 0,1 Kg e capacidade de até $150 \mathrm{Kg}$, a altura, com o estadiômetroSanny ${ }^{\circledR}$ com precisão de $0,5 \mathrm{~cm}$; e a CC, com fita métrica inelástica Sanny ${ }^{\circledR}$ com $1 \mathrm{~cm}$ de largura. Foram utilizados os critérios estabelecidos por Waitzberg e Ferrini, ${ }^{14}$ MarfellJones $^{15}$ e Cameron ${ }^{16}$ para as medidas de peso, altura e CC, respectivamente.

A classificação do estado nutricional foi realizada por meio do Índice de Massa Corporal $\left(\mathrm{IMC}=\right.$ Peso/Altura $\left.{ }^{2}\right)$, preconizado pela Organização Mundial de Saúde (OMS) ${ }^{17}$ Esta variável foi categorizada em IMC $<25 \mathrm{Kg} / \mathrm{m}^{2}$ (sem excesso de peso) e IMC $\geq 25 \mathrm{Kg} / \mathrm{m}^{2}$ (com excesso de peso).

Quanto à circunferência de cintura, foram adotados os pontos de corte preconizados pelo National Cholesterol Education Program's - Adult Treatment Painel III (NCEP - ATP-III). ${ }^{18}$ Dessa forma, foram classificados com elevado risco cardiovascular os indivíduos que apresentaram circunferências da cintura maior ou igual a $102 \mathrm{~cm}$ para homens e maior ou igual a $88 \mathrm{~cm}$ para mulheres.

Com relação às características de trabalho, foram determinadas as seguintes categorias: cargo/função desempenhado atualmente, tempo como policial, considerar o trabalho estressante, considerar-se um profissional valorizado e se sentir realizado em seu trabalho. A variável cargo foi categorizada em agente, delegado/escrivão, investigador e outros. A variável tempo como policial foi categorizada em até 2 anos, entre 2 e 10 anos e mais de 10 anos. Por sua vez, as variáveis "considerar o trabalho estressante", "considerar-se um profissional valorizado" e "se sentir realizado em seu trabalho" foram categorizadas em "sim" e "não".

Os entrevistados foram questionados sobre os seguintes hábitos alimentares: local onde realizam refeições, frequência com que realizam o café da manhã fora de casa, motivação para a composição do prato, consumo de frituras, hábito de utilizar temperos completos e volume de água ingerido diariamente.

O modelo teórico utilizado neste estudo está apresentado na figura 1. 


\section{BLOCO 1}

\section{Características sociodemográficas}

(Sexo, faixa etária, escolaridade, estado civil, raça/cor, classe econômica, renda familiar líquida)

\section{BLOCO 2}

\section{Caracteristicas de trabalho}

(Cargo/função, tempo como policial, considerar o trabalho estressante, considerar-se um profissional valorizado, se sentir realizado com o trabalho)

\section{BLOCO 3}

\section{Hábitos alimentares}

(Motivação na composição do prato, local onde realiza refeições, consumo de frituras, uso de temperos completos, realizar o café da manhã fora de casa, volume de água ingerido diariamente)

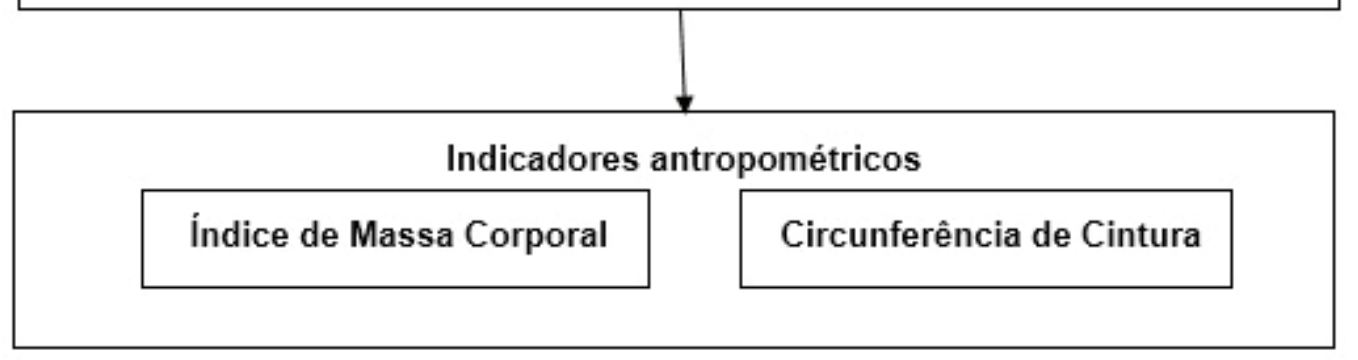

Figura 1. Modelo teórico hierarquizado das possíveis relações entre variáveis sociodemográficas, caraterísticas de trabalho, hábitos alimentares e indicadores antropométricos de policiais civis. Vitória (ES), Brasil, 2014-2015.

Para descrever as variáveis de estudo, foram utilizadas medidas de tendência central (média e mediana), e para as variáveis contínuas, medidas de dispersão (desvios-padrão e erro-padrão da média), e percentuais para as variáveis categóricas. Para a análise das diferenças das proporções, utilizou-se o teste do qui-quadrado $\left(\mathrm{X}^{2}\right)$. O nível de significância foi estabelecido em $\propto 0,05$. Nos casos de significância estatística de até $20 \%$ no teste qui-quadrado, calculou-se o odds ratio ajustado 
pelo modelo de regressão logística multivariada, considerando a categoria de eutrofia para IMC e circunferência da cintura adequada como padrão. O cálculo do odds ratio ajustado foi estimado para as categorias sexo, faixa etária, classificação econômica, cargo/função, tempo como policial, considerar o trabalho estressante, motivação na composição do prato, hábito de realizar o café da manhã fora de casa e volume de água ingerido diariamente. As análises estatísticas foram feitas com o programa SPSS for Windows, versão 18.0.

\section{Resultados}

Dos 134 policiais sorteados, 133 completaram o estudo. A caracterização sociodemográfica e antropométrica da população estudada revelou que dos 133 policiais, 70,7\% $(\mathrm{n}=94)$ eram do sexo masculino, 88,7\% ( $\mathrm{n}=118)$ da amostra pertencia às classes econômicas C, D ou E e 80,5\% ( $\mathrm{n}=107)$ da população apresentou renda familiar líquida superior a $\mathrm{R} \$ 5.000,00$. A maioria dos avaliados possuía mais de 40 anos $(70,8 \%, \mathrm{n}=92)$ e a média de idade foi de $44,27 \pm 9,87$ anos. Quanto ao estado civil, observou-se que a maioria $(65,2 \%, \mathrm{n}=86)$ era casada ou vivia com companheiro. Em relação à escolaridade, constatou-se elevado nível entre os policiais, uma vez que 58\% ( $\mathrm{n}=76$ ) apresentaram nível superior/universitário completo e 23,7\% ( $\mathrm{n}=31$ ), pós-graduação.

A avaliação antropométrica revelou que $64,3 \%(\mathrm{n}=83)$ dos policiais apresentaram excesso de peso, sendo a média de IMC $26,91 \pm 4,36 \mathrm{Kg} / \mathrm{m}^{2}\left(19,36-45,20 \mathrm{Kg} / \mathrm{m}^{2}\right)$. Por sua vez, a análise da CC encontrou $21,1 \%(\mathrm{n}=27)$ de policiais com valor acima do considerado adequado, sendo a média encontrada de $89,33 \pm 12,01 \mathrm{~cm}$.

Observou-se associação entre indicadores antropométricos e variáveis sociodemográficas e de trabalho (Tabela 1). Apresentaram-se associadas ao IMC as variáveis sexo $(\mathrm{p}=0,0010)$, tempo como policial $(p=0,036)$ e o considerar o trabalho estressante $(p=0,001)$. Em relação ao sexo, os homens apresentaram maior prevalência de excesso de peso $(83,1 \%, \mathrm{n}=69)$, quando comparado às mulheres. Quanto às variáveis de trabalho, percebeu-se que quanto maior o tempo de trabalho, maior a ocorrência de excesso de peso, sendo este mais prevalente entre os indivíduos com mais de 10 anos de profissão $(57,8 \%, \mathrm{n}=48)$. Profissionais que consideraram o trabalho estressante também revelaram maiores prevalências de excesso de peso $(87,8 \%, \mathrm{n}=72)$ quando comparados àqueles que não consideram o trabalho estressante $(58,7 \%, \mathrm{n}=27)$. Com relação à circunferência da cintura, apenas a variável faixa etária mostrou associação significativa. Policiais com 40 anos ou mais apresentaram circunferência da cintura mais elevada quando comparado aos policias com menos de 40 anos $(93,3 \%, \mathrm{n}=26)$. 
Tabela 1. Indicadores do Estado Nutricional e suas associações com variáveis sociodemográficas e de trabalho em policiais civis da Grande Vitória (ES), Brasil, 2014-2015.

\begin{tabular}{|c|c|c|c|c|c|c|c|c|}
\hline \multirow{4}{*}{ Variáveis } & \multicolumn{4}{|c|}{ Índice de Massa Corporal } & \multicolumn{4}{|c|}{ Circunferência da Cintura } \\
\hline & & Sem & Com & & & & & \\
\hline & $\begin{array}{c}\text { TOTAL } \\
\mathbf{n}(\%)^{\mathrm{a}}\end{array}$ & $\begin{array}{c}\text { excesso de } \\
\text { peso }\end{array}$ & $\begin{array}{c}\text { excesso de } \\
\text { peso }\end{array}$ & $\mathrm{p}$-valor ${ }^{\mathrm{b}}$ & $\begin{array}{c}\text { TOTAL } \\
\text { n }(\%)^{\mathrm{a}}\end{array}$ & $\begin{array}{c}\text { Adequada } \\
\mathrm{n}(\%)\end{array}$ & $\begin{array}{c}\text { Elevada } \\
\text { n }(\%)\end{array}$ & $\mathrm{p}$-valor ${ }^{\mathrm{b}}$ \\
\hline & & $\mathrm{n}(\%)$ & $\mathrm{n}(\%)$ & & & & & \\
\hline \multicolumn{9}{|l|}{ Sexo } \\
\hline Feminino & $37(28,5)$ & $23(48,9)$ & $14(16,9)$ & \multirow{2}{*}{$0,000^{c}$} & $37(28,7)$ & $27(26,5)$ & $10(37,0)$ & \multirow{2}{*}{0,280} \\
\hline Masculino & $93(71,5)$ & $24(51,1)$ & $69(83,1)$ & & $92(71,3)$ & $75(73,5)$ & $17(63,0)$ & \\
\hline \multicolumn{9}{|l|}{ Faixa etária } \\
\hline$\leq 40$ anos & $37(28,7)$ & $18(39,1)$ & $19(22,9)$ & \multirow{2}{*}{0,051} & $37(28,9)$ & $36(35,6)$ & $1(3,7)$ & \multirow{2}{*}{$0,001 * * *$} \\
\hline$>40$ anos & $92(71,3)$ & $28(60,9)$ & $64(77,1)$ & & $91(71,1)$ & $65(64,4)$ & $26(93,3)$ & \\
\hline \multicolumn{9}{|l|}{ Classific. (ABEP) } \\
\hline $\mathrm{A}$ e B & $15(11,5)$ & $3(6,4)$ & $12(14,5)$ & \multirow{2}{*}{0,166} & $15(11,6)$ & $11(10,8)$ & $4(14,8)$ & \multirow{2}{*}{0,561} \\
\hline C, D e E & $115(88,5)$ & $44(93,6)$ & $71(85,5)$ & & $114(88,4)$ & $91(89,2)$ & $23(85,2)$ & \\
\hline \multicolumn{9}{|l|}{ Raça/Cor } \\
\hline Branca & $61(47,3)$ & $22(47,8)$ & $39(47,0)$ & \multirow{2}{*}{0,927} & $61(47,7)$ & $48(47,5)$ & $13(48,1)$ & \multirow{2}{*}{0,954} \\
\hline Não branca & $68(52,7)$ & $24(52,2)$ & $44(53,0)$ & & $67(52,3)$ & $43(52,5)$ & $14(51,9)$ & \\
\hline \multicolumn{9}{|l|}{ Escolaridade } \\
\hline $1^{\circ}$ e $2^{\circ}$ grau & $22(16,9)$ & $5(10,6)$ & $17(20,5)$ & \multirow{4}{*}{0,489} & $21(16,3)$ & $13(12,7)$ & $8(29,6)$ & \multirow{4}{*}{0,125} \\
\hline Superior & $73(56,2)$ & $29(61,7)$ & $44(53)$ & & $73(56,6)$ & $61(59,8)$ & $12(44,4)$ & \\
\hline Pós-graduado & $31(23,8)$ & $12(25,5)$ & $19(22,9)$ & & $31(24)$ & $24(23,5)$ & $7(25,9)$ & \\
\hline Sem informação & $4(3,1)$ & $1(2,1)$ & $3(3,6)$ & & $4(3,1)$ & $4(3,9)$ & $0(0,0)$ & \\
\hline \multicolumn{9}{|l|}{ Estado Civil } \\
\hline Solteiro & $31(24,0)$ & $14(30,5)$ & $17(20,5)$ & \multirow{3}{*}{0,209} & $31(24,2)$ & $27(26,7)$ & $4(14,8)$ & \multirow{3}{*}{0,300} \\
\hline Casado/mora junto & $85(65,9)$ & $26(56,5)$ & $59(71,1)$ & & $85(66,4)$ & $66(65,3)$ & $19(71,4)$ & \\
\hline Divorciado/ viúvo & $13(10,1)$ & $6(13,0)$ & $7(8,4)$ & & $12(9,4)$ & $8(7,9)$ & $4(14,8)$ & \\
\hline \multicolumn{9}{|l|}{ Renda Familiar } \\
\hline$\leq \mathrm{R} \$ 5.000$ & $25(19,2)$ & $11(23,9)$ & $13(15,7)$ & \multirow{2}{*}{0,247} & $24(18,8)$ & $21(20,8)$ & $3(11,1)$ & \multirow{2}{*}{0,252} \\
\hline$>\mathrm{R} \$ 5.000$ & $105(80,8)$ & $35(76,1)$ & $70(84,3)$ & & $104(81,2)$ & $80(79,2)$ & $24(88,9)$ & \\
\hline \multicolumn{9}{|l|}{ Cargo/função } \\
\hline Investigador & $61(46,0)$ & $16(34,0)$ & $45(54,2)$ & \multirow{4}{*}{0,172} & $60(46,5)$ & $44(43,1)$ & $16(59,3)$ & \multirow{4}{*}{0,330} \\
\hline Delegado/escrivão & $11(8,5)$ & $5(10,6)$ & $6(7,2)$ & & $11(8,5)$ & $8(7,8)$ & $3(11,1)$ & \\
\hline Agente & $19(14,6)$ & $8(17,0)$ & $11(13,3)$ & & $19(14,7)$ & $17(16,7)$ & $2(7,4)$ & \\
\hline Outros & $39(20,0)$ & $18(38,3)$ & $21(25,3)$ & & $39(30,3)$ & $33(32,4)$ & $6(22,2)$ & \\
\hline
\end{tabular}




\begin{tabular}{|c|c|c|c|c|c|c|c|c|}
\hline \multirow[b]{2}{*}{ Variáveis } & \multicolumn{4}{|c|}{ Índice de Massa Corporal } & \multicolumn{4}{|c|}{ Circunferência da Cintura } \\
\hline & $\begin{array}{l}\text { TOTAL } \\
\mathbf{n}(\%)^{\mathrm{a}}\end{array}$ & $\begin{array}{c}\text { Sem } \\
\text { excesso de } \\
\text { peso } \\
\mathrm{n}(\%)\end{array}$ & $\begin{array}{c}\text { Com } \\
\text { excesso de } \\
\text { peso } \\
\text { n }(\%)\end{array}$ & $\mathrm{p}$-valor ${ }^{\mathrm{b}}$ & $\begin{array}{c}\text { TOTAL } \\
\text { n }(\%)^{\mathrm{a}}\end{array}$ & $\begin{array}{c}\text { Adequada } \\
\text { n (\%) }\end{array}$ & $\begin{array}{c}\text { Elevada } \\
\text { n (\%) }\end{array}$ & $\mathrm{p}$-valor ${ }^{\mathrm{b}}$ \\
\hline Tempo como $\mathrm{p}$ & & & & & & & & \\
\hline$<2$ anos & $28(21,5)$ & $16(34,0)$ & $12(14,5)$ & & $28(21,7)$ & $26(25,5)$ & $2(7,4)$ & \\
\hline 2 a 10 anos & $34(26,2)$ & $11(23,4)$ & $23(27,7)$ & $0,036^{\mathrm{c}}$ & $34(26,4)$ & $30(29,4)$ & $4(14,8)$ & 0,09 \\
\hline$>10$ anos & $68(52,3)$ & $20(42,6)$ & $48(57,8)$ & & $67(51,9)$ & $46(45,1)$ & $21(77,8)$ & \\
\hline $\begin{array}{l}\text { Considera trab } \\
\text { estressante }\end{array}$ & & & & & & & & \\
\hline $\begin{array}{l}\text { Sim } \\
\text { Não }\end{array}$ & $\begin{array}{l}99(77,5) \\
29(22,5)\end{array}$ & $\begin{array}{l}27(58,7) \\
19(41,3)\end{array}$ & $\begin{array}{l}72(87,8) \\
10(12,2)\end{array}$ & $0,001^{c}$ & $\begin{array}{l}99(78,0) \\
28(22,0)\end{array}$ & $\begin{array}{l}48(76,2) \\
15(23,8)\end{array}$ & $\begin{array}{l}51(79,7) \\
13(20,3)\end{array}$ & 0,635 \\
\hline $\begin{array}{l}\text { Considera-se } \\
\text { um profissiona } \\
\text { valorizado }\end{array}$ & & & & & & & & \\
\hline $\begin{array}{l}\text { Sim } \\
\text { Não }\end{array}$ & $\begin{array}{l}51(39,8) \\
77(60,2)\end{array}$ & $\begin{array}{l}20(43,5) \\
26(56,5)\end{array}$ & $\begin{array}{l}31(37,8) \\
51(62,2)\end{array}$ & 0,529 & $\begin{array}{l}51(40,2) \\
76(59,8)\end{array}$ & $\begin{array}{l}42(42,0) \\
58(58,0)\end{array}$ & $\begin{array}{c}9(33,3) \\
18(66,7)\end{array}$ & 0,415 \\
\hline $\begin{array}{l}\text { Se sente realiza } \\
\text { seu trabalho }\end{array}$ & & & & & & & & \\
\hline $\begin{array}{l}\text { Sim } \\
\text { Não }\end{array}$ & $\begin{array}{l}94(73,4) \\
34(26,6)\end{array}$ & $\begin{array}{l}33(71,7) \\
13(28,3)\end{array}$ & $\begin{array}{l}61(74,4) \\
21(25,6)\end{array}$ & 0,792 & $\begin{array}{l}93(73,2) \\
34(26,8)\end{array}$ & $\begin{array}{l}72(72,0) \\
28(28,0)\end{array}$ & $\begin{array}{l}21(77,8) \\
6(22,2)\end{array}$ & 0,547 \\
\hline
\end{tabular}

a Total de indivíduos igual a 130, valores diferentes indicam perda de dados.; ${ }^{\mathrm{b}}$ Teste qui-quadrado; ${ }^{\mathrm{c}} \mathrm{p}<0,05$

Os mesmos indicadores se mostraram associados às variáveis relativas aos hábitos alimentares dos policiais (Tabela 2). Quanto ao IMC, foram observadas associações com a motivação na composição do prato $(\mathrm{p}=0,013)$, hábito de realizar o café da manhã fora de casa $(\mathrm{p}=0,035)$ e volume de água ingerido diariamente ( $\mathrm{p}=0,041)$. A CC não se mostrou associada aos hábitos alimentares investigados. Aqueles indivíduos que se motivavam pelo sabor da refeição apresentaram maiores prevalências de excesso de peso $(55,4 \%, \mathrm{n}=46)$, segundo IMC, quando comparados aos que se motivavam pela presença do alimento saudável $(44,6 \%, \mathrm{n}=37)$. Da mesma forma, policiais que possuíam como hábito realizar o café da manhã fora de casa mais que 4 vezes na semana apresentaram maiores prevalências de excesso de peso quando comparados àqueles que realizavam em casa $(20,5 \%, \mathrm{n}=17)$. Menores prevalências de excesso de peso foram observadas naqueles indivíduos que ingeriam 2 litros ou mais de água por dia, em comparação com aqueles que consumiam quantidades inferiores $(62,2 \%, \mathrm{n}=31)$. 
Tabela 2. Indicadores do Estado Nutricional e suas associações com variáveis relativas ao hábito alimentar em policiais civis da Grande Vitória (ES), Brasil, 2014-2015.

\begin{tabular}{|c|c|c|c|c|c|c|c|c|}
\hline \multirow{3}{*}{ Variáveis } & \multicolumn{4}{|c|}{ Índice de Massa Corporal } & \multicolumn{4}{|c|}{ Circunferência da cintura } \\
\hline & Total* & $\begin{array}{c}\text { Sem } \\
\text { excesso } \\
\text { de peso }\end{array}$ & $\begin{array}{c}\text { Com } \\
\text { excesso } \\
\text { de peso }\end{array}$ & $\mathrm{p}$-valor ${ }^{\mathrm{b}}$ & Total $^{\mathrm{a}}$ & Adequada & Elevada & $\mathrm{p}$-valor ${ }^{\mathrm{b}}$ \\
\hline & n $(\%)$ & n (\%) & n $(\%)$ & & n $(\%)$ & $\mathrm{n}(\%)$ & n (\%) & \\
\hline \multicolumn{9}{|l|}{$\begin{array}{l}\text { Motivação na } \\
\text { composição do prato }\end{array}$} \\
\hline $\begin{array}{l}\text { Sabor } \\
\text { Alimento saudável }\end{array}$ & $\begin{array}{l}61(47,3) \\
68(52,7)\end{array}$ & $\begin{array}{l}15(32,6) \\
31(67,4)\end{array}$ & $\begin{array}{l}46(55,4) \\
37(44,6)\end{array}$ & $0,013^{c}$ & $\begin{array}{l}61(47,7) \\
67(53,3)\end{array}$ & $\begin{array}{l}46(45,5) \\
55(54,5)\end{array}$ & $\begin{array}{l}15(55,6) \\
12(44,4)\end{array}$ & 0,355 \\
\hline \multicolumn{9}{|l|}{$\begin{array}{l}\text { Local onde realiza } \\
\text { refeições }\end{array}$} \\
\hline $\begin{array}{l}\text { Em casa } \\
\text { Fora de Casa }\end{array}$ & $\begin{array}{l}63(48,8) \\
66(51,2)\end{array}$ & $\begin{array}{l}25(54,3) \\
21(45,7)\end{array}$ & $\begin{array}{l}38(45,8) \\
45(54,2)\end{array}$ & 0,351 & $\begin{array}{l}63(49,2) \\
65(50,8)\end{array}$ & $\begin{array}{l}52(51,5) \\
49(48,5)\end{array}$ & $\begin{array}{l}11(40,7) \\
16(59,3)\end{array}$ & 0,321 \\
\hline \multicolumn{9}{|l|}{ Consumo de frituras } \\
\hline $\begin{array}{l}\text { Sim } \\
\text { Não }\end{array}$ & $\begin{array}{l}85(66,4) \\
43(33,6)\end{array}$ & $\begin{array}{l}30(65,2) \\
16(34,8)\end{array}$ & $\begin{array}{l}55(67,1) \\
27(32,9)\end{array}$ & 0,831 & $\begin{array}{l}43(33,9) \\
84(66,1)\end{array}$ & $\begin{array}{l}32(32,0) \\
68(68,0)\end{array}$ & $\begin{array}{l}11(40,7) \\
15(59,2)\end{array}$ & 0,394 \\
\hline \multicolumn{9}{|l|}{$\begin{array}{l}\text { Uso de temperos } \\
\text { completos }\end{array}$} \\
\hline $\begin{array}{l}\text { Sim } \\
\text { Não }\end{array}$ & $\begin{array}{l}29(77,5) \\
100(22,5)\end{array}$ & $\begin{array}{l}9(19,6) \\
37(80,4)\end{array}$ & $\begin{array}{l}20(24,1) \\
63(75,9)\end{array}$ & 0,555 & $\begin{array}{l}29(22,7) \\
99(77,3)\end{array}$ & $\begin{array}{l}24(23,8) \\
77(76,2)\end{array}$ & $\begin{array}{l}5(18,5) \\
22(81,5)\end{array}$ & 0,563 \\
\hline \multicolumn{9}{|l|}{$\begin{array}{l}\text { Realizar café da } \\
\text { manhã fora de casa }\end{array}$} \\
\hline $1-4 \mathrm{x}$ semana & $16(12,4)$ & $9(19,6)$ & $7(8,4)$ & & $16(12,5)$ & $13(12,9)$ & $3(11,1)$ & \multirow{4}{*}{0,252} \\
\hline$>4 \mathrm{x}$ na semana & $20(15,5)$ & $3(6,5)$ & $17(20,5)$ & $0,035^{\mathrm{c}}$ & $20(15,6)$ & $13(12,9)$ & $7(25,9)$ & \\
\hline Nunca/quase nunca & $93(72,1)$ & $34(73,9)$ & $59(63,4)$ & & $92(71,9)$ & $75(74,3)$ & $17(63,0)$ & \\
\hline \multicolumn{8}{|l|}{$\begin{array}{l}\text { Volume de água } \\
\text { ingerido diariamente }\end{array}$} & \\
\hline Menos de 2L & $71(55,5)$ & $20(43,5)$ & $51(62,2)$ & \multirow[b]{2}{*}{$0,041^{c}$} & $70(55,1)$ & $56(56,0)$ & $14(51,9)$ & \multirow[b]{2}{*}{0,701} \\
\hline 2L ou mais & $57(44,5)$ & $26(56,5)$ & $31(37,8)$ & & $57(44,9)$ & $44(44,0)$ & $13(48,1)$ & \\
\hline
\end{tabular}

\footnotetext{
a Total de indivíduos igual a 130 , valores diferentes indicam perda de dados.; ${ }^{\mathrm{b}}$ Teste qui-quadrado; ${ }^{\mathrm{c}} \mathrm{p}<0,05$
} 
Após as análises de regressão logística (Tabela 3), mantiveram-se associados ao IMC as variáveis sexo $(p=0,007)$, a classe socioeconômica $(p=0,032)$, considerar o trabalho estressante $(p=0,000)$ e hábito de realizar o café da manhã fora de casa $(\mathrm{p}=0,015)$. Por sua vez, apenas a faixa etária apresentou-se associada à circunferência da cintura $(\mathrm{p}=0,010)$.

No grupo de variáveis sociodemográficas, ser do sexo masculino (Odds 5,23; IC 1,57-17,42) e pertencer às classes econômicas A e B (Odds 8,18; IC 1,20-55,86) aumentou as chances de o indivíduo apresentar IMC inadequado. Com relação à circunferência da cintura, possuir mais de 40 anos elevou em 14,4 vezes as chances de ter CC elevada (IC 1,88-110,57). Em relação às variáveis de trabalho, considerar o trabalho estressante aumentou em 11,17 vezes a chance de apresentar IMC inadequado (IC 3,09-40,36). Quanto ao hábito alimentar, aqueles policiais que realizavam o café da manhã fora de casa com frequência superior a 4 vezes na semana apresentaram uma chance 10,68 vezes maior de possuir IMC inadequado (IC 1,57-72,67).

Tabela 3. Fatores sociodemográficos, características do trabalho e hábitos alimentares associados aos indicadores do estado nutricional, a partir do OddsRatio ajustado, considerando a categoria Índice de Massa Corporal e Circunferência da Cintura adequados como padrão, em policiais civis da Grande Vitória (ES), Brasil, 2014-2015.

\begin{tabular}{|c|c|c|c|c|c|c|c|c|c|c|}
\hline \multirow{3}{*}{ 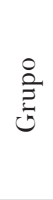 } & \multirow{3}{*}{ Variáveis } & \multicolumn{9}{|c|}{ Índice de massa corporal } \\
\hline & & \multirow{2}{*}{$\begin{array}{c}\text { Qui- } \\
\text { quadrado } \\
\text { p-valor }\end{array}$} & \multicolumn{4}{|c|}{ OddsRatio bruto } & \multicolumn{4}{|c|}{ OddsRatio ajustado (a) } \\
\hline & & & $\mathrm{p}$-valor & OR & LI $95 \%$ & LS 95\% & p-valor & OR & LI $95 \%$ & LS 95\% \\
\hline \multirow{3}{*}{1} & Sexo Masculino & 0,001 & 0,001 & 4,72 & 2,10 & 10,62 & 0,007 & 5,23 & 1,57 & 17,42 \\
\hline & $\begin{array}{l}\text { Faixa etária acima de } \\
40 \text { anos }\end{array}$ & 0,051 & 0,053 & 2,17 & 0,99 & 4,74 & 0,889 & 0,88 & 0,15 & 5,13 \\
\hline & $\begin{array}{l}\text { Classificação econômica } \\
\text { A e B }\end{array}$ & 0,166 & 0,178 & 2,48 & 0,66 & 9,28 & 0,032 & 8,18 & 1,20 & 55,86 \\
\hline \multirow{3}{*}{2} & Cargo/função Investigador & 0,172 & 0,042 & 2,41 & 1,03 & 5,64 & 0,964 & 0,97 & 0,25 & 3,77 \\
\hline & $\begin{array}{l}\text { Tempo como policial mais } \\
\text { de } 10 \text { anos }\end{array}$ & 0,036 & 0,012 & 3,20 & 1,29 & 7,97 & 0,099 & 3,32 & 0,80 & 13,81 \\
\hline & $\begin{array}{l}\text { Considera trabalho } \\
\text { estressante }\end{array}$ & 0,001 & 0,000 & 5,07 & 2,09 & 12,27 & 0,000 & 11,17 & 3,09 & 40,36 \\
\hline
\end{tabular}




\begin{tabular}{|c|c|c|c|c|c|c|c|c|c|c|}
\hline \multirow{3}{*}{ 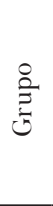 } & \multirow{3}{*}{ Variáveis } & \multicolumn{9}{|c|}{ Índice de massa corporal } \\
\hline & & \multirow{2}{*}{$\begin{array}{c}\text { Qui- } \\
\text { quadrado } \\
\text { p-valor }\end{array}$} & \multicolumn{4}{|c|}{ OddsRatio bruto } & \multicolumn{4}{|c|}{ OddsRatio ajustado (a) } \\
\hline & & & $\mathrm{p}$-valor & OR & LI $95 \%$ & LS $95 \%$ & $\mathrm{p}$-valor & OR & LI $95 \%$ & LS 95\% \\
\hline \multirow{3}{*}{3} & $\begin{array}{l}\text { Motivação na composição } \\
\text { do prato sabor }\end{array}$ & 0,060 & 0,062 & 2,02 & 0,97 & 4,23 & 0,081 & a & a & a \\
\hline & $\begin{array}{l}\text { Realizar café da manhã } \\
\text { fora de casa }>4 \text { vezes por } \\
\text { semana }\end{array}$ & 0,035 & 0,074 & 3,27 & 0,89 & 11,96 & 0,015 & 10,68 & 1,57 & 72,67 \\
\hline & $\begin{array}{l}\text { Volume de água ingerido } \\
\text { diariamente }\end{array}$ & 0,041 & 0,042 & 2,14 & 1,03 & 4,46 & 0,235 & a & a & a \\
\hline \multirow{3}{*}{ 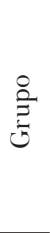 } & & \multicolumn{9}{|c|}{ Circunferência de cintura } \\
\hline & Variáveis & $\begin{array}{l}\text { Qui- } \\
\text { quadrado }\end{array}$ & \multicolumn{4}{|c|}{ OddsRatio bruto } & \multicolumn{4}{|c|}{ OddsRatio ajustado (a) } \\
\hline & & $\mathrm{p}$-valor & p-valor & OR & LI $95 \%$ & LS $95 \%$ & $\mathrm{p}$-valor & OR & LI $95 \%$ & LS 95\% \\
\hline 1 & $\begin{array}{l}\text { Faixa etária acima de } 40 \\
\text { anos }\end{array}$ & 0,001 & 0,010 & 14,40 & 1,88 & 110,57 & 0,010 & 14,40 & 1,88 & 110,57 \\
\hline 2 & $\begin{array}{l}\text { Tempo como policial mais } \\
\text { de } 10 \text { anos }\end{array}$ & 0,090 & 0,022 & 5,93 & 1,29 & 27,35 & 0,273 & a & a & a \\
\hline
\end{tabular}

Modelo de Regressão logística, hierárquico (segundo os grupos), ajustado pelo método forward razão de verossimilhança. a: variáveis retiradas do modelo pelo método de ajuste de variáveis.

LI: Limite inferior; LS: Limite superior; OR: Oddsratio; em negrito: variáveis estatisticamente significantes ao nível de $5 \%$.

\section{Discussão}

O presente estudo evidenciou alta prevalência de excesso de peso entre os policiais civis avaliados $(64,3 \% ; \mathrm{n}=83)$. Além disso, identificou que ser do sexo masculino (OR 5,23; IC 1,5717,42), pertencer à classe socioeconômica mais elevada (A e B) (OR 8,18; IC 1,20-55,86), considerar o trabalho estressante (OR 11,17, IC 3,09-40,36) e realizar o desjejum fora de casa (OR 10,68, IC 1,57-72,67) são fatores de risco para o aumento do IMC, e pertencer à faixa etária acima de 40 anos, para a elevação da circunferência da cintura (OR 14,40, IC 1,88-110,57).

Tais resultados reforçam a estreita relação entre variáveis sociodemograficas, ocupacionais e hábitos alimentares e o peso corporal. Ademais, evidenciam que o excesso de peso também é elevado em categorias profissionais consideradas fisicamente ativas e nas quais o condicionamento físico é importante para o desempenho da função. ${ }^{19}$ 
O elevado número de profissionais com excesso de peso (64,3\%) está bastante acima da média estimada em 2014 para a população brasileira pela Vigilância de Fatores de Risco e Proteção para Doenças Crônicas por Inquérito Telefônico (VIGITEL) (52,8\%) e para a população de Vitória/ES (51\%). ${ }^{3}$ Essa realidade também foi encontrada em estudos avaliando policiais civis e militares ${ }^{10,20,21,22}$ de diferentes estados brasileiros, onde se verificou taxas de excesso de peso iguais ou superiores à $60 \%$ na população investigada. Considerando as maiores exigências física a que esta categoria está exposta e a necessidade de condicionamento físico para execução de algumas atividades, esperava-se menor prevalência de excesso de peso nessa população.

É possível supor que a alta taxa de sobrepeso observada entre os policiais esteja relacionada com a prática de atividade física insuficiente, decorrente da própria característica peculiar da profissão e seus reflexos sobre a vida pessoal desses policiais, expostos a condições precárias de trabalho, sobrecarga emocional, inversão dos horários de sono e cargas horárias excessivas, que fazem os policiais aproveitarem suas folgas para descansar, realizando atividades de lazer com menos gasto de energia. ${ }^{23}$

O percentual de excesso de peso foi maior entre os indivíduos do sexo masculino. Um estudo realizado com 34 indivíduos, adultos e idosos de ambos os sexos, em Belém (PA) corrobora o achado, uma vez que foi verificado que 90,0\% dos homens e 64,3\% das mulheres apresentavam sobrepeso e obesidade segundo o IMC. ${ }^{21}$ Da mesma forma, um importante inquérito nacional indica a evolução do perfil antropométrico no Brasil, evidenciando o aumento da prevalência de excesso de peso entre os homens em todas as grandes regiões do país. ${ }^{24} \mathrm{O}$ predomínio de policiais do sexo masculino na faixa de sobrepeso pode se justificar pela constante preocupação feminina com questões estéticas, sua maior percepção dos riscos à saúde e o desenvolvimento de hábitos alimentares normalmente mais saudáveis do que o dos homens. ${ }^{21}$ Deve-se destacar que o excesso de peso representa prejuízos para saúde, tendo em vista a forte relação com doenças cardiovasculares, diabetes, dislipidemias, alta mortalidade ${ }^{2}$ e consequente prejuízo para a qualidade de vida desse grupo de servidores. Além disso, a obesidade pode reduzir a mobilidade desses profissionais, sua aptidão física e desempenho necessário à execução das atividades de combate, além de dificultar a utilização de coletes balísticos, coldres e fardamentos, o que, por sua vez, pode colocar em risco a saúde e segurança do próprio policial.

Deve-se considerar que os homens tendem a apresentar maior proporção de gordura abdominal, conferindo-lhes o chamado padrão masculino ou androide de distribuição de gordura corporal; por sua vez, mulheres têm maior quantidade de gordura na região glútea, apresentando o padrão feminino ou ginoide. ${ }^{25}$ Apesar disso, o sexo não se mostrou associado à circunferência da cintura na presente investigação.

A prevalência de sobrepeso e obesidade se acentua com a idade, atingindo um valor maior na faixa etária de 45 a 54 anos. ${ }^{26}$ A idade é fator adicional para o desenvolvimento de problemas de 
saúde e o denominado "envelhecimento funcional precoce", que pode atingir os trabalhadores ainda em idade produtiva. ${ }^{27}$ No entanto, apenas a circunferência da cintura mostrou-se associada à faixa etária neste estudo.

A associação entre IMC inadequado e classe econômica elevada também foi observada por outros pesquisadores envolvendo outros grupos populacionais. ${ }^{28,29}$ Considerava-se que indivíduos de baixa classe socioeconômica apresentariam proteção natural contra o excesso de peso, uma vez que esse grupo tenderia a ter uma menor disponibilidade de alimentos, logo, menor consumo energético, e maior intensidade de atividade física relacionada à ocupação. ${ }^{28,30}$ No entanto, ao analisarmos a prevalência de excesso de peso entre os países desenvolvidos, e, portanto, de maior renda quando comparados aos países em desenvolvimento, verifica-se que o excesso de peso não está associado à renda per capta do país, tendo em vista que, apesar de os Estados Unidos ainda apresentarem o maior percentual de excesso de peso, países como México, Chile, Turquia também estão entre os com maior percentual de obesidade segundo dados apresentados pela Organização para a Cooperação e o Desenvolvimento Econômico (OCDE). ${ }^{31}$

$\mathrm{Na}$ amostra estudada, o maior risco de os policiais das classes A e B apresentarem IMC inadequado pode estar relacionado às funções predominantemente administrativas que cargos de maior remuneração apresentam, fato este que levaria ao menor gasto energético decorrente da execução dessas atividades quando comparado às de combate.

Com relação à escolaridade, diferente dos dados da presente pesquisa, as complicações de saúde em policiais foram associadas à baixa escolaridade. ${ }^{10,32}$ Apesar disso, estudos têm demonstrado que o baixo grau de conhecimento em nutrição está associado aos desvios nutricionais em populações de baixa renda, ${ }^{33}$ e o nível de escolaridade tem sido apontado como variável capaz de interferir na forma como a população escolhe seus alimentos, que pode ser decisiva para a qualidade do autocuidado e para a capacidade de interpretar informações relativas à proteção da saúde. ${ }^{34}$ Segundo Teichmann e colaboradores, ${ }^{35}$ esta associação pode ser atribuída, em parte, aos efeitos de pouca informação a que está submetida a população de baixa escolaridade, à adoção de um estilo de vida menos saudável e consequente aumento do consumo de alimentos de baixo custo e alta densidade energética. Dessa forma, acredita-se que a educação é capaz de influenciar o conhecimento sobre alimentação e nutrição que, por sua vez, relaciona-se ao estado nutricional dos indivíduos. ${ }^{36}$

A idade apresentou importante relação com estado nutricional, sendo maior a prevalência de CC inadequada entre os policias com idade superior a 40 anos. Estudos mostram que, com o passar dos anos, o indivíduo perde grande quantidade de massa magra e acumula maior quantidade de massa gorda, principalmente na região abdominal, levando ao aumento do risco de diversas patologias, entre elas hipertensão arterial e doenças cardiovasculares. ${ }^{37}$ 
A profissão policial civil é considerada estressante, visto a necessidade do contato contínuo entre agente e sociedade no desenvolvimento da função, já que este desempenha seu ofício em um ambiente conflitivo, no limite da marginalidade e criminalidade. ${ }^{38}$ Assim sendo, o presente estudo confirma os achados na literatura, uma vez que, aliado ao estresse profissional, o estilo de vida sedentário propicia o crescimento das taxas de obesidade e sobrepeso nesse grupo, que segue o perfil nacional.,39

Em obesos existe uma forte tendência para o aumento da ativação fisiológica induzida pelo estresse, que se manifesta na presença de desafios ambientais, como o estresse crônico ocupacional. ${ }^{40}$ Ademais, obesos têm o hábito de se alimentar muito rapidamente em substituição à irritabilidade advinda do trabalho, além de comer mais quando estão estressados. ${ }^{41}$

O risco relacionado ao estresse laboral reforça indícios de que esta variável pode ser desencadeadora de manifestações psicológicas, como depressão e ansiedade. Acredita-se que a ansiedade estaria ligada a uma maior ingestão calórica, podendo chegar, em alguns casos, à compulsão alimentar ou "bingeeating", extremamente relacionada com o início do processo de aumento da massa corporal. ${ }^{42}$

$\mathrm{Na}$ atualidade, tem crescido o número de brasileiros que fazem suas refeições fora do lar, ${ }^{39}$ e entre elas, o café da manhã. A maior chance de policiais que realizam o café da manhã fora de casa na maioria dos dias da semana apresentarem IMC inadequado pode ser devido à maior densidade energética das preparações ofertadas fora do lar - e entre elas, o café da manhã -, que são, de forma geral, mais ricas em gorduras totais, gorduras saturadas, açúcares, e pobres em micronutrientes. ${ }^{43}$

Nesse sentido, corroborando o achado, foi observado que essa modalidade de alimentação está associada à maior prevalência de excesso de peso e sedentarismo. ${ }^{44}$ Além disso, sobrepeso e obesidade são maiores em homens que realizam refeições fora do lar $(38,5 \%$ e $11,9 \%$, respectivamente) quando comparados aos que não o fazem $\left(36,1 \%\right.$ e 10,3\%). ${ }^{45}$ É importante ressaltar que são escassos os estudos que avaliam a qualidade nutricional dessas refeições no Brasil.

De acordo com a circunferência de cintura, detectou-se um número importante de policiais civis com elevado risco de complicações metabólicas e coronárias associadas ao excesso de peso e gordura visceral. Um estudo realizado no Paraná, encontrou um percentual de $12 \%$ (n = 22) de policiais nas mesmas condições. ${ }^{22}$ No Rio Grande de Sul, em uma investigação com 112 policiais militares, 18,27\% $(\mathrm{n}=19)$ da amostra apresentava excesso de gordura na região abdominal. ${ }^{21}$ Esses achados sugerem que as altas taxas de excesso de peso e circunferência de cintura elevada observadas na população avaliada estejam relacionadas com a prática de atividade física insuficiente, hábitos alimentares inadequados e estresse laboral. 
As informações obtidas neste estudo poderão acrescentar à literatura médica brasileira dados acerca do estado nutricional, hábitos alimentares e caracterização sociodemografica e ocupacional de policiais civis, atualmente pouco investigados e disponíveis.

Deve-se sublinhar que limitações metodológicas são comuns aos estudos realizados em corporações policiais de todo o mundo, e que no Brasil esse fato se torna mais evidente visto as restrições ao acesso à informação e o receio das corporações de que o repasse de informações de ordem pessoal prejudique os entrevistados. ${ }^{10}$ Por este motivo, o viés de informação deve ser destacado como uma possível limitação deste artigo. Outro ponto limitante no estudo é seu delineamento transversal, que impossibilita a determinação de relação temporal entre as variáveis estudadas. Ressalta-se, também, o fato de as respostas ao questionário serem autorreferidas, o que possibilita a subestimação ou superestimação de dados. Além disso, como esta investigação se dá com uma categoria profissional especifica, a generalização dos resultados é restrita. Outra limitação a ser destacada é que se utilizou neste estudo o IMC como parâmetro para avaliação do peso. Este parâmetro, por sua vez, não difere entre massa muscular e massa gorda, devendo-se, por esta razão, analisar com cautela o percentual de excesso de peso encontrado. Para minimizar esta limitação, também foi utilizada a CC para parâmetro alternativo de avaliação antropométrica.

\section{Considerações finais}

A partir do presente estudo, evidencia-se a importância da avaliação antropométrica em policiais civis, a fim de identificar possíveis fatores associados à inadequação do estado nutricional nesse grupo de servidores, permitindo-nos concluir que a inadequação dos indicadores do estado nutricional tem relação direta com classe econômica, idade, sexo, estresse laboral e hábito alimentar. Dessa forma, este estudo fornece dados para subsidiar ações de promoção à saúde e qualidade de vida nesse grupo laboral.

\section{Referências}

1. Malta DC, Moura L, Prado RR, Escalante JC, Schmidt MI, Duncan DD. Mortalidade por doenças crônicas não transmissíveis no Brasil e suas regiões, 2000 a 2011. Epidemiol Serv. Saúde 2014; 23(4):599-608.

2. Goulart FAA. Doenças crônicas não transmissíveis: estratégias de controle e desafios e para os sistemas de saúde. Brasília: Organização Pan-Americana da Saúde, Organização Mundial da Saúde; 2012. 92 p.

3. Brasil. Ministério da Saúde. Secretaria de Vigilância em Saúde. VIGITEL BRASIL 2013: vigilância de fatores de risco e proteção para doenças crônicas por inquérito telefônico. Brasília: Ministério da Saúde; 2014. 120 p. 
4. World Health Organization. Obesity: Preventing and managing the global epidemic. Report. Geneva: WHO; 1997. (WHO technical report series, 894)

5. Vasques ACJ, Priore SE, Rosado LEFP, Franceschini SCC. Utilização de medidas antropométricas Para a Avaliação do acúmulo de Gordura visceral. Rev. Nutr. 2010; 23(1):107-118.

6. Popkin BM. Contemporary nutritional transition: determinants of diet and its impact on body composition. Proc Nutr Soc. 2011; 70(1):82-91.

7. Wandel M, Roos G. Work, food and physical activity. A qualitative study of coping strategies among men in three occupations. Appetite 2005; 44(1):93-102.

8. Bezerra IN, Sichieri R. Characteristics and spending on out-of-home eating inBrazil. Rev Saude Pública 2010; 44(2):221-229.

9. Martinez MC, Paraguay AIBB. Satisfação e saúde no trabalho, aspectos conceituais e metodológicos. Cadernos de Psicologia Social do Trabalho 2003; 6:59-78.

10. Minayo MCS, Assis SG, Oliveira RVC. Impacto das atividades profissionais na saúde física e mental dos policiais civis e militares do Rio de Janeiro. Ciênc. Saúde Coletiva 2011; 16(4):2199-209.

11. Silva MD, Simeão Júnior CA. Fatores associados à obesidade em policiais militares. FDeportes.com. Revista Digital. Buenos Aires [Internet] 2014; 18(188). Disponível em: http://www.efdeportes.com/ efd188/fatores-associados-a-obesidade-em-policiais-militares.htm.

12. Minayo MCS, ASSIS SG, Oliveira, RVC. Impacto das atividades profissionais na saúde física e mental dos policiais civis e militares do Rio de Janeiro (RJ, Brasil). Ciênc. Saúde Coletiva 2011; 16(4):2199-2209.

13. Associação Brasileira de Empresas de Pesquisa (ABEP). Critério de Classificação Econômica Brasil (CCEB), 2013 [Internet]. Disponível em: http://www.abep.org/criterio-brasil

14. Waitzberg DL, Ferrini, MT. Exame físico e antropometria. In: Waitzberg DL. Nutrição oral, enteral e parenteral na prática clínica. São Paulo: Atheneu; 2000. p. 255-278.

15. Stewart A, Marfell-Jones M. International standards for anthropometric assessment. ISAK: Potchefstroom; 2006.

16. Cameron N. The measurement of human growth. Sydney: Croom Helm; 1984.

17. World Health Organization. Obesity: preventing and managing the global epidemic. Report of a World Health Organization Consultation. Geneva: WHO; 2000. 256 p.

18. Expert Panel on Detection, Evaluation, and treatment of high blood cholesterol in adults. Executive summary of the third report of the National Cholesterol Education Program (NCEP) Expert Panel on Detection, Evaluation, and Treatment of High Blood Cholesterol in Adults (Adult Treatment Panel III). JAMA 2001; 285:2486-2497.

19. Mazini Filho ML, Silva AC, Venturine GRO, Aidar FJ, Rodrigues BM, et al. Avaliação do condicionamento físico de policiais militares da $146^{a}$ companhia especial de Policia Militar. Revista Brasileira de Prescrição e Fisiologia do Exercício 2012; 6(35):486-493.

20. Ferreira SKD, Bonfim C, Augusto SGL. Fatores associados ao estilo de vida de Policiais Militares. Ciênc Saúde Coletiva 2011; 16(8):3403-3412. 
21. Barbosa RO, Silva EF. Prevalência de fatores de risco cardiovascular em policiais militares. Revista Brasileira de Cardiologia 2013; 26(1):45-53.

22. Donadussi C, Oliveira AF, Fatel ECS, Dichi JB, Dichi I. Ingestão de lipídios na dieta e indicadores antropométricos de adiposidade em policiais militares. Rev. Nutr. 2009; 22(6):847-855.

23. Turuchima MT, Nascimento T, Bennemann RM. Associação entre indicadores antropométricos em relação ao risco para doenças cardiovasculares. Saúde e Pesquisa 2015; 8(Edição Especial):55-63.

24. Instituto Brasileiro de Geografia e Estatística. Pesquisa de orçamentos familiares 2008-2009: análise do consumo alimentar pessoal no Brasil. Rio de Janeiro: IBGE; 2011. 150 p.

25. Dagenais GR, Yi Q, Mann JF, Bosch J, Pogue J, Yusuf S. Prognostic impact of body weight and abdominal obesity in women and men with cardiovascular disease. Am Heart J. 2005; 149(1):54-60.

26. Pinheiro ARO, Freitas SFT, Corso ACT. Uma abordagem epidemiológica da obesidade. Rev Nutr. 2004; 17(4):523-533.

27. Moreno CRC, Louzada FM. What happens to the body when one works at night? Cad Saúde Pública 2004; 20(6):1739-1745.

28. Monteiro CA, Conde WL. Tendência secular da desnutrição e da obesidade na infância na cidade de São Paulo. Rev Saúde Pública 2000; 34(6):52-61.

29. Silva VS. Prevalência de sobrepeso/obesidade e fatores associados em adultos no Brasil [dissertação]. Florianópolis (SC): Universidade Federal de Santa Catarina; 2010.

30. Sobal J, Stunkard AJ. Socioeconomic status and obesity: a review of the literature. Psychol Bull. 1989; 105(2):260-275.

31. Organisation for Economic Co-operation and Development (OECD). Obesity Update [Internet]. jun. 2014. [acesso em 03 jul. 2016]. Disponível em: http://www.oecd.org/health/Obesity-Update-2014.pdf.

32. Ferreira, SKD. Condições de saúde, de trabalho e modos de vida de policiais militares: estudo de caso na Cidade do Recife - PE. 2009. [tese] Recife: Centro de Pesquisas Aggeu Magalhães; 2009. 202 f.

33. Rodrigues CB, Batista JS, Lustosa IB, Diniz DB. Inter-relação entre as práticas alimentares e o grau de conhecimento em nutrição das famílias residentes em duas comunidades de risco em Fortaleza, CE. Revista de APS 2012; 15(1):35-41.

34. Velásquez-Meléndez G, Pimenta AM, Kac G. Epidemiologia do sobrepeso e da obesidade e seus fatores determinantes em Belo Horizonte (MG), Brasil: estudo transversal de base populacional. Rev Panam Salud Publica 2004; 16(5): 308-314.

35. Teichmann L, Olinto MTA, Costa JSD, Ziegler D. Fatores de risco associados ao sobrepeso e a obesidade em mulheres de São Leopoldo, RS. Rev Bras Epidemiol. 2006; 9(3):360-973.

36. Dattilo M, Furlanetto P, Kuroda AP, Nicastro H, Coimbra PCFC, Simony RF. Conhecimento nutricional e sua relação com índice de massa corporal. Nutrire 2009; 34(1):75-84.

37. Tumerelo S, Santos Júnior MF, Nunes NCR. Influência da idade sobre os valores de pressão arterial e frequência cardíaca em repouso. Revista Digital efdeportes.com [Internet] 2003; 9(60). Disponível em: http://www.efdeportes.com/efd60/repouso.htm 
38. Sanchez-Milla JJ, Sanz-Bou MA, Apellaniz-Gonzalez A, Pascual-Izaola A. Policia y estrés laboral. Estressores organizativos como causa de morbilidad psiquiátrica. Revista de la Sociedad Española de Salud Laboral em la Administración Pública SESLAP 2001; 1(4):21-25.

39. Instituto Brasileiro de Geografia e Estatística. Pesquisa de Orçamentos Familiares 2008-2009: Antropometria e Estado Nutricional de Crianças, Adolescentes e Adultos no Brasil. Rio de Janeiro: IBGE; 2010.

40. Steptoe A, Cropley M, Griffith J, Joekes K. The influence of abdominal obesity and chronic work stress on ambulatory blood pressure in men and women. Int J Obes Relat Metab Disord. 1999; 23(11):1184-91.

41. Nishitani N, Sakakibara H, Akiyama I. Eating behavior related to obesity and job stress in male Japanese workers. Nutrition 2009; 25(1):45-50.

42. Maranhão Neto GA, Miranda CJM. Detecção do risco de sobrepeso em servidoras universitárias: um estudo exploratório. Revista Brasileira de Fisiologia do Exercício 2003; 2(2):166-171.

43. Kant AK, Graubard BI. Eating out in América, 1987 - 2000: trends and nutritional correlates. Prev Med. 2004; 38:243-9. 19.

44. Orfanos P, Naska A, Trichopoulos D, et al. Eating out of home and is correlates in 10 European countries. The European Prospective Investigation into Cancer and Nutrition (EPIC) study. Public Health Nutr. 2007; 10:1515-1525.

45. Bezerra IN, Sichieri R. Eating out of home and obesity: a Brazilian Nationwide survey. Public Health Nutrition 2009; 12(11):2037-2043. 